\title{
THE EFFECT OF CONTROL SIGNALS TIME DELAYS ON THE TRAJECTORY OF A DISPERSED GROUP OF ROBOTS
}

\author{
M. Siwek ${ }^{*}$, L. Baranowski**, J. Panasiuk ${ }^{* * *}$, W. Kaczmarek ${ }^{\dagger}$, K. Besseghieur ${ }^{\ddagger}$
}

\begin{abstract}
The main aim of this article is to present the effect of control signal time delays on the trajectory of a group of robots with a dispersed structure. The research was carried out for a group of three robots with the same structure. The results for the assumed delay range are presented in the form of comparison of the reference trajectory with the delayed one and the distance between robots during the movement.
\end{abstract}

Keywords: mobile robot, trajectory of dispersed group of robots, time delay in mobile robotic

\section{Introduction}

The development of new technologies is related to the need for faster transmission and increasing amounts of data. The observed development trend is optical fiber optic technology, which allows to achieve much higher transmission speeds than with other transmission methods. However, taking into account the willingness of the network users to achieve mobility, the Wi-Fi transmission remains an inseparable element. At a time when we observe a very large increase in the purchase and use of mobile devices such as laptops, tablets and smartphones, it can be said that wireless communication is most often used by an ordinary user. Due to many advantages of wireless transmission, this type of communication is also the most commonly used in mobile robotics. This allows the robot to achieve full mobility both indoor and in the open space. Wireless transmission is used to send device control data as well as feedback data, e.g. image from cameras, sound, environmental data, measured and collected by sensors integrated in the robot. However, wireless transmission is not without flaws. Particular attention should be paid to environments in which there are many Wi-Fi networks, e.g. office buildings, factories, large clusters of devices generating many disturbances and causing damping. In the case of wireless communication in such places one should expect data transmission errors and signals delays. The delay in data transmission is a significant problem in the control of the robot (swarm) group (Vazquez-Santacruz et al., 2017) Significant delay of the control data can cause inaccuracies in trajectories of the robot group, which may be critical for the correct performance of the robot task. In the case of a robot group organized in the hierarchical structure, where each entity must stay in the continuous contact with others, the signal delay has a significant impact on making decisions by the control algorithm and assigning tasks to members of the group. The solution of the control delay problem may stem from the use of the structure of a dispersed group of robots and time synchronization at various stages of the task.

\footnotetext{
* Michał Siwek, MS.: Faculty of Mechatronics \& Aerospace, Millitary University of Technology, Kaliskiego 2, 00-908, Warsaw, PL, michal.siwek@wat.edu.pl

** Prof. Leszek Baranowski, PhD.: Faculty of Mechatronics \& Aerospace, Millitary University of Technology, Kaliskiego 2, 00-908, Warsaw, PL, leszek.baranowski@wat.edu.pl

*** Jarosław Panasiuk, PhD.: Faculty of Mechatronics \& Aerospace, Millitary University of Technology, Kaliskiego 2, 00-908, Warsaw, PL, jaroslaw.panasiuk@wat.edu.pl

† Wojciech Kaczmarek, PhD.: Faculty of Mechatronics \& Aerospace, Millitary University of Technology, Kaliskiego 2, 00908, Warsaw, PL, wojciech.kaczmarek@wat.edu.pl

‡ Khadir Besseghieur, MS.: Faculty of Mechatronics \& Aerospace, Millitary University of Technology, Kaliskiego 2, 00-908, Warsaw, PL, besseghieurkh@hotmail.fr
} 


\section{The object of the research}

Controlling the dispersed group of robots, i.e. the robot swarm is based on building a common network by the robots included in the group. These robots interpret, independently from each other, the received information from the management center. Based on the individual analysis of the received data robots take action and change their positions in order to correctly perform the assumed task. Regardless of the configuration and reconfiguration of the swarm, the implementation of each task must be carried out in a synchronous manner by all robots belonging to the specific groups, which have been assigned the specific tasks (Takahashi, 2004).

The object of the research described in the article involves a group composed of three two-wheeled robot. In order to simplify an analysis of the single robot motion, a model of a replacement (with one drive wheel of replacement) has been introduced. This wheel is situated in the geometric center of the robot. Assuming that the movement takes place within one plane, there are imposed nonholonomic constraints associated with the coordinates of the geometrical center of the robot $\mathrm{x}_{\mathrm{S}}$ and $\mathrm{y}_{\mathrm{S}}$ as shown in (3).Mathematical models of robots are based on (Giergiel et al., 2002, Wei and Huilin, 2011) and is shown by the system of differential equations:

$$
\begin{aligned}
& r\left(2 m_{z}+m_{r}\right)[\ddot{\varphi} \cos \theta-\dot{\varphi} \dot{\theta} \sin \theta]=\lambda_{1} \\
& r\left(2 m_{z}+m_{r}\right)[\ddot{\varphi} \sin \theta-\dot{\varphi} \dot{\theta} \cos \theta]=\lambda_{2} \\
& \left(2 m_{r} d^{2}+I_{R z}+2 I_{K x}+2 I_{K z} h^{2}\right) \ddot{\theta}=\left(M_{L}-M_{R}-N_{L} f_{L}+N_{R} f_{R}\right) h \\
& {\left[\left(2 m_{z}+m_{r}\right) r^{2}+2 I_{K z}\right] \ddot{\varphi}=M_{L}+M_{R}-N_{L} f_{L}-N_{R} f_{R}} \\
& \dot{x}_{S}-r \dot{\varphi} \cos \theta=0, \quad \dot{y}_{S}-r \dot{\varphi} \sin \theta=0
\end{aligned}
$$

where: $\mathrm{r}$ - radius of wheels; $\mathrm{m}_{\mathrm{z}}$ - weight of the replacement wheel; $\mathrm{m}_{\mathrm{r}}$ - weight of the robot structure; $\mathrm{I}_{\mathrm{Rz}}$ - moment of inertia of the structure relative to the $\mathrm{Z}$ axis; $\mathrm{I}_{\mathrm{Kz}} \mathrm{I}_{\mathrm{Kx}}$ - the moment of inertia of the replacement wheel relative to the $\mathrm{Z}$ axis and the $\mathrm{X}$ axis; $\varphi$ - rotation angle of wheels; $\theta$ - robot orientation angle in the global coordinate system; $d$ - track of wheel; $N_{L}, N_{R}$ - pressure forces of the left and right wheels; $f_{L}, f_{R}-$ coefficients of friction of the left and right wheels on the ground; $h=\frac{r}{d}$; $\lambda_{1}, \lambda_{2}$-Lagrange multiplier.

While building a distributed structure of a group of robots it should be assumed, that the coordinates of the individual robots are calculated independently of each other, but in reference to the common stationary coordinate system. In order to perform the assumed tasks in a proper way, the following necessary steps must be taken: formulation of the initial conditions (deployment of the robots) and time synchronization for all robots. During the task the robot swarm can change its configuration, divide into
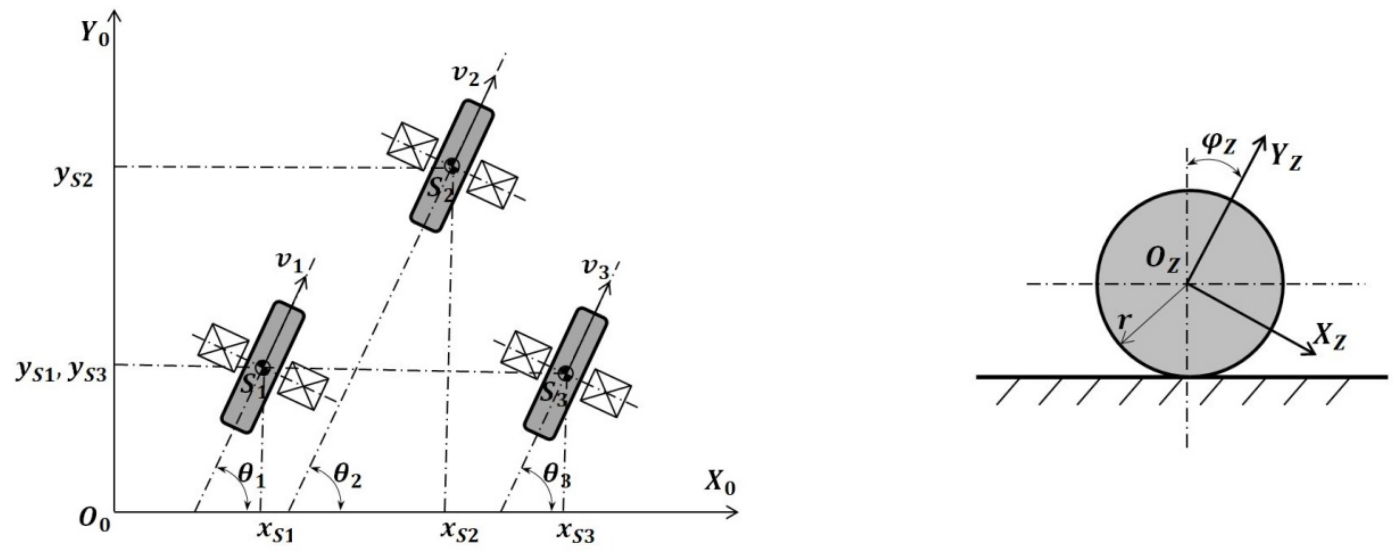

Fig. 1: Example of a dispersed configuration of a group of robots (simplification of robots for a model with one substitute wheel). 
smaller swarms or compose a big swarm out of the several small swarms. The implementation of each task must be done in a synchronous manner by all involved robots. For this purpose, a very important point is to send control commands, which can be picked up by robots without time delays. An exemplary configuration of a dispersed group of robots (simplified to a model with one replacement wheel) is shown in Fig. 1.

Using the assumptions concerning the control of the dispersed group of robots a simulation system (in the Matlab Simulink environment) has been developed. The system enables to study how the control delays imposed on the individual robots affect the movement of the whole group. The structure of the system in the form of a block diagram is shown in Fig. 2.

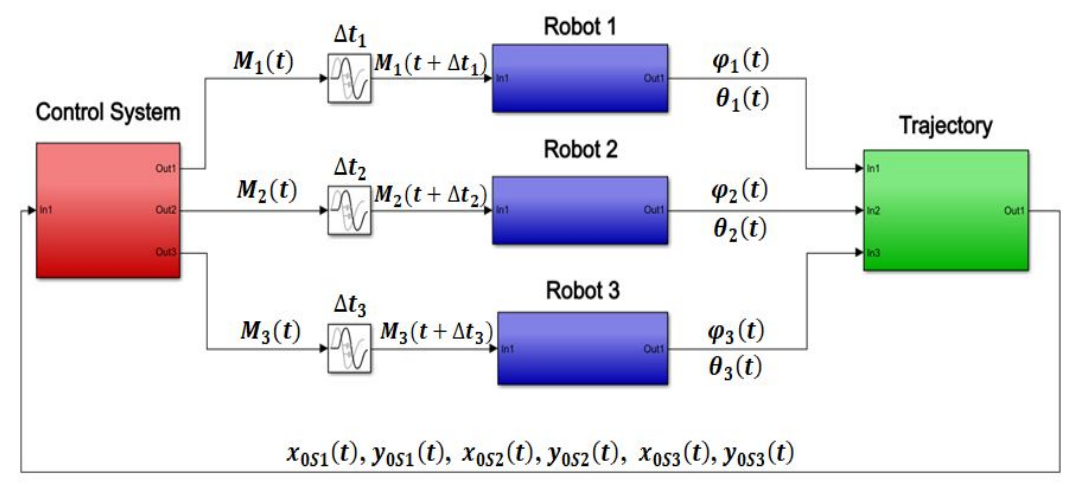

Fig. 2: Block diagram of a robot group motion simulation system.

\section{Research}

In order to study the effect of time delays in motion control signals, robots were set into a group forming an equilateral triangle with a side length of $1 \mathrm{~m}$. The geometric centers of the robots were placed in the vertices of the triangle.

The tests were carried out for random values of the delay of the control signal ranging from 300 to 3000 [ms]. The range of delay values has been selected on the basis of delay tests for various types of network connections described in (Życzkowski, Stawicki, 2009). Due to the use of a wireless connection in the development of the simulation, the focus was on wireless network models described in this article.

The research was carried out for two control scenarios:

- passing the set distance by a straight line and changing the orientation by a given angle during movement (delay on the control signal took place before the start of the move), shown in Fig. 3;

- passing through a straight line and forcing an unexpected change of orientation by a given angle during straight line movement (a sudden change of requirements, delay before the start of the move and before the orientation change), shown in Fig. 4.

The analysis of the movement of groups of robots dispersed structure shows that the time delays have a very significant impact on time and accuracy required to achieve the desired end position and the mutual distances robot during the task. During the first simulation (Fig. 3), the lack of preservation of mutual distance between robots at the beginning of the movement was observed. The difference between the initial and final distances amounted to a few meters. Significant errors were also found for the group configuration after the end of the movement. Group order is not preserved, does not reflect the set of initial configuration of an equilateral triangle. In the case of the second simulation (Fig. 4), there is duplication of errors discussed for the first test as a result of additional control signals appearing during the group motion. In both cases, the delay of the control signal causes significant errors in maintaining the configuration of group of robots and extends the task execution time.

\section{Conclusions}

The article presents the effect of time delays of the control signals on the trajectory of a group of robots with a dispersed structure. The research was carried out for a group of three mobile robots of the same construction. The impact of delays was analyzed for two control scenarios: trajectory planned and sudden change of trajectory. The results show that the time delays have a very significant impact on time and accuracy to achieve the desired end position and on mutual distance between robots during the task. 

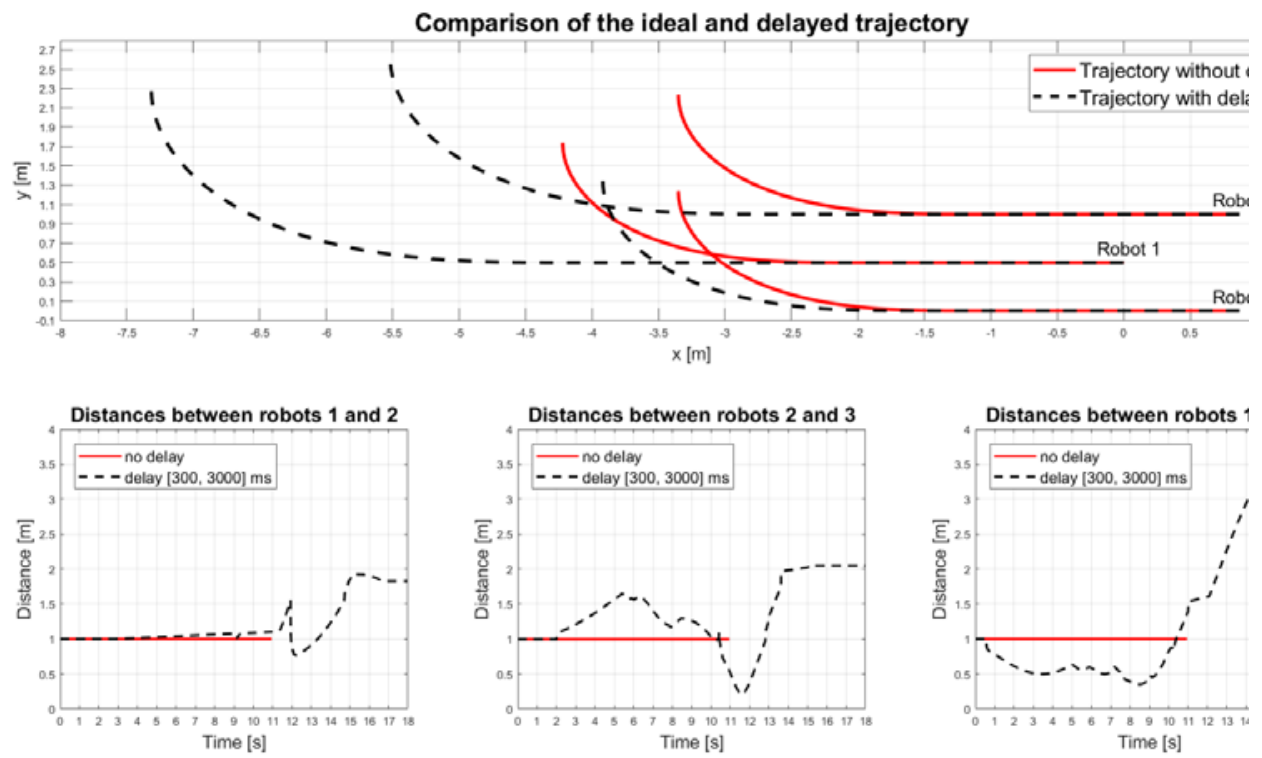

Fig. 3: Experiment 1- drive $2 \mathrm{~m}$ straight and change the orientation by $90^{\circ}$.
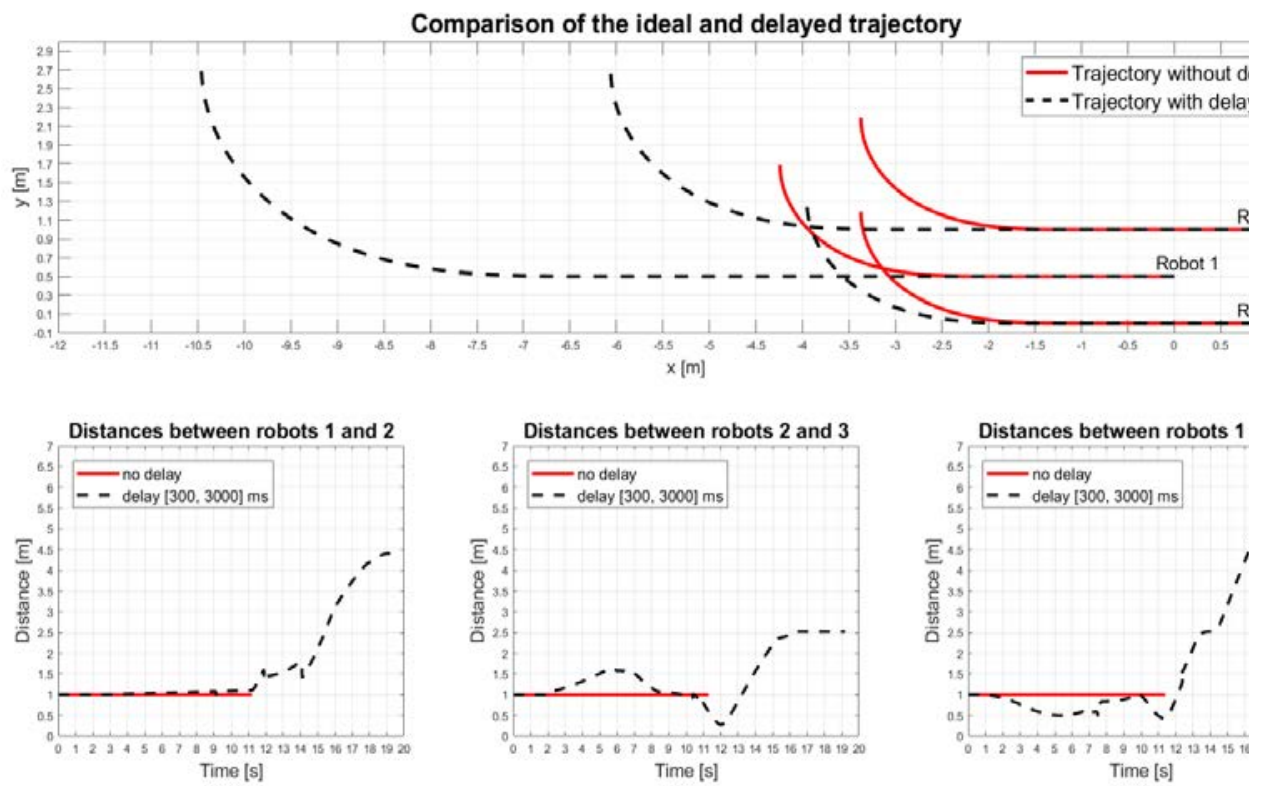

Fig. 4: Experiment 2- linear motion and sudden change of orientation by $90^{\circ}$.

Based on the conducted research, it can be concluded that in the case of distributed control of a group of robots, it is necessary to introduce time synchronization of robots in the control algorithm.

\section{References}

Giergiel M., Hendzel Z., Żylski W. (2002) Modelling and control of mobile wheeled robots, PWN , Warsaw (in Polish).

Takahashi H. (2004) Autonomous decentralized control for formation of multiple mobile robots considering ability of robot, IEEE Trans. On Industrial Electronics, vol. 51, pp.1272-1279.

Vazquez-Santacruz J. A., Velasco-Villa M., Portillo-Velez R. de J., Marin-Urias L. F., Vigueras- Zuniga M. (2017) Autonomous navigation for multiple mobile robots under time delay in communication, Journal of Intelligent \& Robotic Systems, vol. 86, issue 2-4, pp. 583-597.

Wei W., Huilin T. (2011) Reconfigurable multi-robot system kinematic modeling and motion planning, Industrial Electronics and Applications, pp. 1672-1677.

Życzkowski M., Stawicki Ł. (2009) Effect of delays in IP networks on the effectiveness of video monitoring - part 2, ZABEZPIECZENIA 3/2009, pp. 40-48, (in Polish). 